\title{
POURQUOI UNE RELATION POSITIVE ENTRE TAILLE DES EXPLOITATIONS ET PRODUCTIVITÉ AU BURKINA FASO ?
}

Yves Gérard Bazie, Tristan Le Cotty, Élodie Maitre D’hôtel, Damien Oula Ouattara et Audrier Sanou

Société française d'économie rurale | «Économie rurale »

2020/1 n 371 | pages 37 à 58

ISSN 0013-0559

Article disponible en ligne à l'adresse :

https://www.cairn.info/revue-economie-rurale-2020-1-page-37.htm

Distribution électronique Cairn.info pour Société française d'économie rurale.

(c) Société française d'économie rurale. Tous droits réservés pour tous pays.

La reproduction ou représentation de cet article, notamment par photocopie, n'est autorisée que dans les limites des conditions générales d'utilisation du site ou, le cas échéant, des conditions générales de la licence souscrite par votre établissement. Toute autre reproduction ou représentation, en tout ou partie, sous quelque forme et de quelque manière que ce soit, est interdite sauf accord préalable et écrit de l'éditeur, en dehors des cas prévus par la législation en vigueur en France. Il est précisé que son stockage dans une base de données est également interdit. 


\section{Économie rurale}

RURALE

Agricultures, alimentations, territoires

371 | janvier-mars 2020

varia

\section{Pourquoi une relation positive entre taille des exploitations et productivité au Burkina Faso?}

Why is there a positive relationship between farm size and productivity in Burkina Faso?

Yves Gérard Bazie, Tristan Le Cotty, Élodie Maitre D’hôtel, Damien Oula Ouattara et Audrier Sanou

\section{(2) OpenEdition}

Journals

Édition électronique

URL : http://journals.openedition.org/economierurale/7592

DOI : 10.4000/economierurale.7592

ISSN : 2105-2581

Éditeur

Société Française d'Économie Rurale (SFER)

Édition imprimée

Date de publication : 31 mars 2020

Pagination : $37-58$

ISSN : 0013-0559

Distribution électronique Cairn

\section{CAIRN INFO}

CHERCHER, REPÉRER, AVANCER

\section{Référence électronique}

Yves Gérard Bazie, Tristan Le Cotty, Élodie Maitre D'hôtel, Damien Oula Ouattara et Audrier Sanou,

"Pourquoi une relation positive entre taille des exploitations et productivité au Burkina Faso? ?,

Économie rurale [En ligne], 371 | janvier-mars 2020, mis en ligne le 02 janvier 2022, consulté le 30 mars 2020. URL : http://journals.openedition.org/economierurale/7592 ; DOI : https://doi.org/10.4000/ economierurale.7592 


\title{
Pourquoi une relation positive entre taille des exploitations et productivité au Burkina Faso?
}

\author{
Yves Gérard BAZIE • Ministère de l'Agriculture, Ouagadougou, Burkina Faso \\ Tristan LE COTTY, Élodie MAITRE D'HOTEL • CIRAD, Montpellier, France \\ Damien Oula OUATTARA, Audrier SANOU • Ministère de l'Agriculture, Ouagadougou, \\ Burkina Faso
}

La productivité à l'hectare des céréales est plus importante dans les exploitations agricoles de grande taille au Burkina Faso. Cette relation positive va à l'encontre du constat dominant estampillé dans la littérature économique comme la « relation inverse entre taille et productivité ». Cet article analyse l'interdépendance entre les productions de maïs et de coton pour l'accès à l'engrais comme facteur explicatif de la relation positive. Un modèle de production inspiré de Feder (1985) est appliqué à la production du maïs sous contrainte de crédit. II établit que le rendement du maïs augmente avec la surface totale de l'exploitation, à condition que cet accroissement se traduise par une levée de la contrainte de terre cultivable en coton. La validité empirique de ce modèle est testée par la mobilisation de données ménages, de prix et de données pluviométriques au Burkina Faso sur la période 1995-2012.

MOTS-CLÉS : taille, productivité, engrais, Burkina Faso

Why is there a positive relationship between farm size and productivity in Burkina Faso? In Burkina Faso, grain productivity is higher in larger farms. This positive relationship goes against the dominant empirical finding known in the economic literature as the "inverse farm size-productivity relationship." This paper analyzes the interdependence between maize and cotton production for access to fertilizer as an explanatory factor for the positive relationship. A production model inspired by Feder (1985) is applied to the production of maize under credit constraints. It states that maize yield increases with the total area of the farm, provided that this increase translates into a lifting of the cultivable cotton land constraint. The empirical validity of this model is tested using a combination of household, price, and rainfall data for Burkina Faso over the 1995-2012 period. (JEL: Q12).

KEYWORDS: size, productivity, fertilizer, Burkina Faso

\section{Introduction}

\section{Augmentation de la productivité des céréales}

es céréales représentent la majorité de Lla production agricole du Burkina Faso et correspondent aux deux tiers environ de l'apport calorique de la population burkinabè (FAOSTAT data). La production de céréales est donc déterminante dans la sécurité alimentaire et l'augmentation de la productivité ${ }^{1}$ des céréales est un enjeu de développement fort au Burkina Faso. Par rapport à d'autres régions du monde, les rendements moyens en céréales sont faibles. Cette situation s'explique

\footnotetext{
1. Nous utilisons dans cet article indifféremment les termes de productivité et de rendements, la productivité renvoyant à la productivité de la terre et étant mesurée par la production agricole ramenée à la surface.
} 
Figure 1. Évolution de la productivité des céréales au Burkina Faso ( $\mathrm{kg} / \mathrm{ha})$, données du ministère de l'Agriculture du Burkina Faso

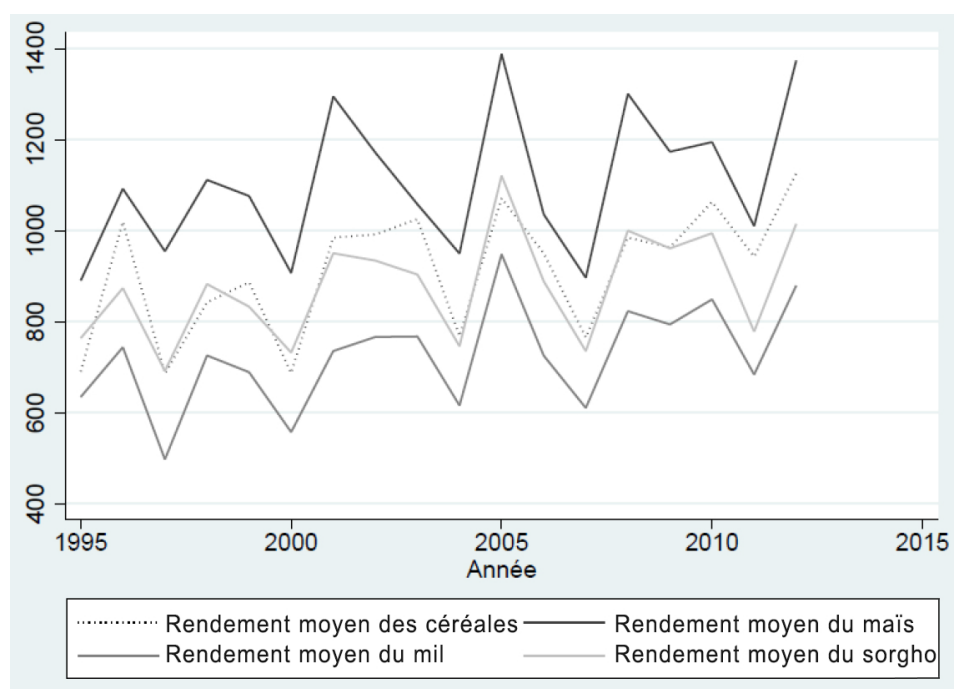

Source : les auteurs.

principalement par un faible niveau de recours aux engrais chimiques (Le Cotty et al., 2018) et par l'existence de sols dégradés au sein desquels nous assistons à des pertes de nutriments sans fertilisation - organique comme minérale - suffisante (Sawadogo et al., 2008). Les rendements moyens en céréales ont cependant sensiblement augmenté au cours des dernières années, comme nous pouvons le voir sur la figure 1. Alors qu'ils s'établissaient au début des années 2000 autour de $800 \mathrm{~kg} / \mathrm{ha}$, ils atteignent aujourd'hui plus de $1000 \mathrm{~kg} / \mathrm{ha}$.

Les céréales sont des cultures pluviales: le niveau de leur production est donc dépendant des pluies, ce qui explique que les rendements réalisés varient fortement d'une année à une autre. Par ailleurs, ces rendements moyens cachent d'importantes variations d'une culture à une autre, d'une région à une autre et d'une exploitation à une autre.

Les rendements moyens nationaux sont d'environ $1200 \mathrm{~kg} / \mathrm{ha}$ pour le maïs,
$900 \mathrm{~kg} / \mathrm{ha}$ pour le sorgho et $800 \mathrm{~kg} / \mathrm{ha}$ pour le mil. Le sorgho et le mil sont deux céréales traditionnelles, qui sont en général produites pour être autoconsommées par les ménages, dont le rendement est très sensible à la pluviométrie et pour lesquelles la principale stratégie d'intensification est l'utilisation de main-d'œuvre plutôt que l'application d'engrais chimique. Le maïs quant à lui est une céréale qui peut être produite pour être autoconsommée par les ménages mais qui est également de plus en plus vendue sur les marchés domestiques et dont la production mobilise l'application d'engrais chimique.

Les régions les plus au nord, situées en zone sahélienne, présentent les plus bas cumuls pluviométriques et enregistrent des rendements de céréales moyens de $600 \mathrm{~kg} / \mathrm{ha}$, alors que les régions situées au sud-ouest du pays, situées en zone soudanienne, bénéficient de cumuls pluviométriques plus avantageux et obtiennent des rendements moyens de céréales de l'ordre de $1500 \mathrm{~kg} / \mathrm{ha}$. 
RECHERCHE

Yves Gérard BAZIE et al.

Enfin, les rendements varient d'une exploitation a une autre, en fonction notamment des caractéristiques des ménages, des systèmes de production agricole choisis, des niveaux d'intensification en engrais chimiques et organiques et de la qualité des sols.

Différents facteurs ont concouru aux gains de rendement observés au cours des deux dernières décennies, et notamment l'augmentation tendancielle des cumuls pluviométriques annuels, la large diffusion de la traction animale, le développement des marchés du maïs et le développement, bien que timide, de l'utilisation de l'engrais chimique.

\section{Relation positive entre taille des exploitations et productivité des céréales}

Il apparaît au Burkina Faso que la productivité des céréales est d'autant plus élevée que la surface des exploitations est grande. Cette relation positive est représentée à la figure 2. Chaque point correspond à une observation de la taille moyenne des exploitations et du rendement moyen des céréales par province et par année. La productivité est exprimée en kilogrammes par hectare et les surfaces sont exprimées en hectares. Le Burkina Faso compte 45 provinces. Il y a 45 provinces et 18 années considérées dans notre analyse. À partir du nuage de points de toutes les observations, nous traçons une droite de régression dont la pente est significativement positive.

Cette relation positive entre la taille moyenne des exploitations et les rendements moyens observés se vérifie également pour la production de maïs. La figure 7 ( $c f$. annexe) présente une pente positive. Cette relation positive se vérifie pour toutes les années étudiées entre 1995 et 2012 et semble même s'accentuer sur la période. La figure 8 ( $c f$. annexe) montre en effet que la pente de la droite de régression est de plus en plus marquée au fil du temps.

Figure 2. Relation entre productivité des céréales et surface totale au Burkina Faso (moyennes par province et par an)

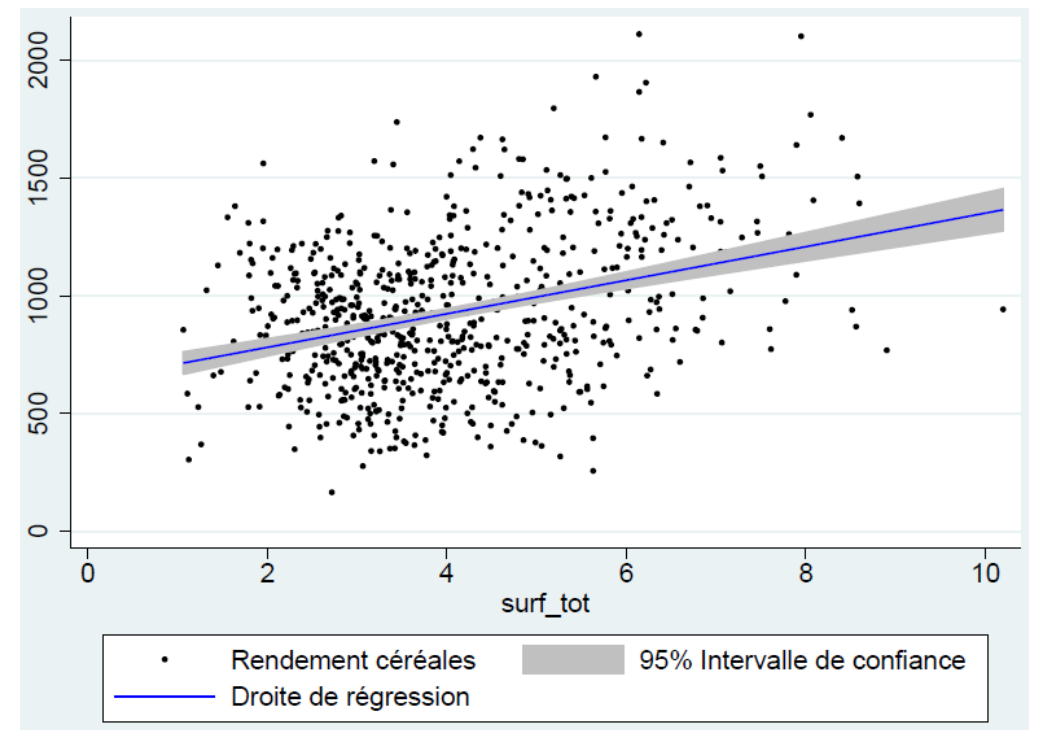

Source : les auteurs. 
Tout semble donc indiquer que, en moyenne provinciale, les plus grandes exploitations sont plus productives au Burkina Faso. Cela va à l'encontre du fait stylisé, souvent confirmé empiriquement, selon lequel les petites exploitations sont plus productives, estampillé dans la littérature économique comme la «relation inverse entre productivité et taille » (Feder, 1985). Des études empiriques anciennes mettent en évidence cette relation négative dans de nombreux pays en développement (Bardhan, 1973 ; Berry et al., 1979) sans toutefois proposer de modèle théorique à même d'expliquer cette relation, notamment sous les hypothèses classiques de fonction de production agricole à rendements constants (pas d'effet direct de la taille sur le rendement) (Berry et al., 1979) et de fonctionnement parfait des marchés (Bhalla et Roy, 1988). Les modèles vont donc s'employer à expliquer l'existence d'une relation négative en relâchant les hypothèses classiques. Les deux principales raisons avancées dans la littérature pour expliquer cette relation négative sont la qualité des sols et les défaillances de marché des facteurs de production (travail, terre, capital) dans les pays en développement (Barrett et al., 2010).

L'argument lié à la qualité des sols énonce que dans l'histoire de l'occupation des sols, les premières exploitations à s'installer l'ont fait sur les terres les plus fertiles (et les suivantes sur les terres moins fertiles) avant de s'agrandir par la mise en culture de terres de moins en moins fertiles. Par conséquent, les plus grandes exploitations présentent des sols plus pauvres (Benjamin, 1995). Le fait que les petites exploitations ont de meilleurs sols (Bhalla, 1988) permet d'expliquer qu'elles obtiennent de meilleurs rendements (Bhalla et Roy, 1988).

L'argument lié aux imperfections de marché concerne à la fois le marché du travail, du crédit (le capital) et celui du foncier (Heltberg, 1998). Dans les pays pauvres, il y a plus de travail familial par hectare sur les petites exploitations parce qu'il n'y a pas de marché du travail permettant d'absorber le surplus de maind'œuvre (Bardhan, 1973). In fine, la production est plus intensive en main-d'œuvre sur les petites exploitations, ce qui se traduit par de meilleurs rendements. Par ailleurs, la défaillance du marché du crédit limite l'achat d'intrants et l'investissement agricole, et donc in fine les gains de rendement (Feder, 1985). Si l'accès au crédit est limité, l'accès au capital pour investir et l'accès aux intrants sont limités également. Ainsi, plus la surface augmente, plus les rapports capital par hectare et intrants par hectare diminuent. Cette relation négative a été mise en évidence et discutée dans de nombreux pays en développement, notamment en Inde (Carter, 1984), à Madagascar (Barrett, 1996) et au Pakistan (Heltberg, 1998). En Afrique, une étude qui associe des données de ménages et de parcelles sur douze pays africains aboutit également à une corrélation négative entre la surface des exploitations et les rendements (Larson et al., 2014).

Cependant, d'autres travaux ont contribué à remettre en cause l'existence d'une relation négative. Feder (1985) a proposé un cadre théorique dans lequel la taille de l'exploitation ne joue sur la productivité agricole que dans le cas où l'exploitation emploie de la main-d'œuvre salariée et où l'effectivité du travail salarié est sensible à la supervision du travail. Dans ce cas, la relation entre la taille et la productivité peut être négative ou positive en fonction du degré de sensibilité du travail à la supervision et de l'élasticité de la production à la taille (hypothèse de production à rendements constants ou non). Ainsi, si les grandes exploitations ont davantage accès au capital et en conséquence peuvent avoir recours a davantage de main-d'œuvre salariée cela ne se traduira par des rendements plus élevés que si les exploitations sont en 
mesure de superviser le travail (Eswaran et Kotwal, 1986).

Sur le plan empirique, des études remettent en question la relation négative entre taille et productivité en mettant en avant une relation positive (Kevane, 1996 ; Collier et Dercon, 2014 ; Zaibet et Dunn, 1998 ; Chand et al., 2011) ou bien l'absence de relation significative (Benjamin, 1995) ou encore en mettant en avant l'existence d'une relation en U (Carter et Wiebe, 1990). Au Kenya par exemple, du fait de l'imperfection des marchés du travail et du crédit, il a été montré que les petites exploitations étaient très intensives en maind'œuvre, mais qu'à l'autre bout du spectre, les grandes exploitations avaient plus de facilités pour accéder au crédit ex ante, ce qui leur permettait également d'obtenir des niveaux élevés de productivité. Une étude conduite à Java montre que la relation négative entre la taille et la productivité ne tient plus lorsque l'on prend en compte les imperfections du marché du travail et la qualité de la terre (Benjamin, 1995). Une étude menée au Soudan aboutit ainsi à une relation positive entre la surface des exploitations et les rendements (Kevane, 1996) et met en avant les contraintes de liquidité comme éléments explicatifs. Selon Kevane (1996), les exploitations les plus riches - les plus grandes - ont moins de contraintes de liquidité et peuvent donc davantage intensifier leur production agricole. Pour Collier et Dercon (2014), des économies d'échelle sont possibles et expliqueraient que dans certains contextes nous ayons une relation positive entre la taille des exploitations et la productivité agricole. Ces économies d'échelle sont réalisables via l'adoption d'innovations technologiques particulières : l'argument présenté est que les grandes exploitations sont dirigées par des personnes plus éduquées et plus susceptibles d'adopter les innovations. Les effets de l'innovation sont insensibles à la taille (par exemple, il n'y a aucune raison de penser que l'engrais soit plus rentable sur de grandes exploitations que sur de petites exploitations), mais son adoption ne l'est pas, et se fait plus rapidement dans le cas des grandes exploitations (Collier et Dercon, 2014). Des économies d'échelle peuvent également être réalisées dans le domaine du stockage, de la transformation, de la commercialisation des produits agricoles et pourraient expliquer une relation positive entre la taille des exploitations et la productivité agricole (Collier et Dercon, 2014). Cet argument semble corroborer une étude menée en Tunisie dans laquelle les plus grandes exploitations sont davantage orientées vers la commercialisation et présentent de meilleurs rendements (Zaibet et Dunn, 1998). Ces arguments empiriques semblent indiquer que la relation inverse entre productivité et taille pourrait n'être valable que pour une forme d'agriculture traditionnelle mais pas pour les formes d'agriculture commerciale qui financent les facteurs de production fixes et variables (investissement en capital, rémunération de maind'œuvre et achat d'engrais) (Chand et al., 2011 ; Collier et Dercon, 2014).

\section{L'accès à l'engrais via la production de coton comme facteur explicatif potentiel}

Au Burkina Faso, la grande majorité des exploitations agricoles correspond à des formes d'agriculture traditionnelle où la main-d'œuvre est essentiellement familiale. Par ailleurs, les arguments sur la qualité des sols et sur la défaillance des marchés du crédit et du travail semblent plausibles. Ces éléments devraient nous orienter vers une situation dans laquelle la relation entre la taille et le rendement serait négative. L'explication d'une relation positive est donc ailleurs. Notre hypothèse est qu'au Burkina Faso, produire du coton permet de lever la contrainte de crédit et supprime la relation négative attendue entre taille des exploitations et rendement. La production de coton au Burkina Faso 
est encadrée par des sociétés cotonnières qui ont intégré verticalement certaines fonctions et se chargent notamment de la distribution d'engrais chimique à crédit aux producteurs. L'engrais est avancé en début de saison culturale. Il est ensuite remboursé aux sociétés cotonnières en fin de saison via une déduction de son coût sur le prix d'achat du coton aux producteurs. L'engrais distribué par ce mécanisme bénéficie à la fois à la production de coton et à celle de maïs. Historiquement, seule la production de coton bénéficiait de ce système de préfinancement de l'engrais chimique. Cependant, l'engrais était détourné par les producteurs vers la production de maïs, ce qui a engendré des situations de défaut de paiement et a été considéré comme une des raisons de l'inefficacité de la filière coton (Dowd-Uribe, 2014). Pour pallier cette situation, les sociétés cotonnières ont introduit un mécanisme de distribution rationnée de l'engrais pour les productions de coton et de maïs qui dépend des surfaces déclarées en coton. Les quantités d'engrais attribuées sont au maximum de trois sacs de $\mathrm{NPK}^{2}$ pour le coton par ha de coton déclaré et de un sac de NPK pour le maïs par hectare de coton déclaré. Ce qui revient à dire qu'il y a un plafonnement de l'engrais obtenu pour le maïs a un tiers de l'engrais obtenu pour le coton.

Pour se protéger contre le risque de défaut de paiement, des enquêtes terrain sont régulièrement menées pour s'assurer de l'utilisation des engrais obtenus. Le coût des engrais coton et maïs est ensuite déduit de la vente du coton.

La contrainte de crédit, ajoutée à la faiblesse des liquidités financières des ménages agricoles au moment de l'installation des cultures fait que les exploitations sont limitées dans leurs achats d'intrants agricoles et dans leurs décisions

2. Engrais minéral composé d'éléments d'Azote, de Potassium et de Phosphate. d'investissement agricole. Les conséquences sont qu'une augmentation de surface s'accompagne d'une diminution du ratio intrants par hectare et du ratio capital par hectare, et que les petites exploitations sont plus productives que les grandes. Les liquidités financières des ménages agricoles sont limitées au Burkina Faso, surtout à l'installation des cultures. Celleci coöncide avec la période de soudure en Afrique subsaharienne, marquée par l'affaiblissement, voire l'épuisement, des stocks de céréales donc des possibilités d'accès aux liquidités financières pour les ménages agricoles. L'accès au crédit rural est par ailleurs très limité au Burkina Faso. La frilosité des institutions financières à se positionner en milieu rural tient aux nombreux risques qui pèsent sur l'activité agricole et à la faiblesse des garanties financières dont disposent les ménages agricoles. L'accès au crédit est cependant plus développé dans la zone soudanienne au travers du crédit engrais fourni par les sociétés cotonnières pour la production de coton et de maïs. L'engrais est distribué à crédit pour le coton (trois sacs de NPK par hectare de coton déclaré) et pour le maiis (un sac de NPK par hectare de coton déclaré). Le nombre de sacs d'engrais obtenus pour le maïs est plafonné (supra) à un tiers des sacs obtenus pour le coton. $\mathrm{Ce}$ qui revient à dire que la surface de maïs financée par le crédit intrant correspond au maximum à un tiers de la surface de coton financée ${ }^{3}$.

Plus la surface cultivée en coton est grande, plus l'accès à l'engrais à crédit pour la production de maïs est grand et permet de lever la contrainte de liquidité. La quantité d'engrais fournie à crédit par

3. À titre d'illustration numérique, un producteur déclarant 3 hectares de coton recevra 12 sacs de NPK : 9 sacs pour le coton - qu'il appliquera sur ses 3 ha de coton - ainsi que 3 sacs pour le maïs, qu'il appliquera sur 1 ha de maïs. 
les sociétés cotonnières dépend directement de la surface cultivée en coton.

L'idée principale défendue dans cet article est que produire du coton au Burkina Faso permet aux producteurs agricoles de lever les contraintes de liquidité et de crédit. Ces contraintes sont d'autant plus « levées » que les surfaces déclarées en coton sont importantes.

Le reste de l'article est organisé comme suit. La deuxième partie présente un modèle d'exploitation agricole adapté de Feder (1985) pour tenir compte de la complémentarité des productions de coton et de maïs au Burkina Faso. Le modèle estime la demande d'engrais du producteur agricole et intègre deux fonctions de production liées, pour le maïs et pour le coton. Le modèle aboutit à une relation de causalité entre la surface totale et le rendement du maïs. La troisième partie teste la validité empirique d'une telle relation, à partir de la combinaison de bases de données sur les prix, sur les cumuls pluviométriques et sur les caractéristiques des ménages et des exploitations agricoles au Burkina Faso. Les données correspondantes à la période 1995 à 2012 couvrent l'ensemble du territoire du Burkina Faso (45 provinces) et sont traitées en panel par la méthode des moments généralisés.

\section{Un modèle de production agricole avec rationnement d'engrais}

\section{Le maïs et le coton, deux productions liées}

Chez Feder, le travail salarié est le principal facteur de production, mais dans notre cas, la quantité d'engrais utilisée est le principal facteur de production de maïs, dont nous cherchons à expliquer l'utilisation. Dans notre analyse, nous considérons deux productions, le maïs et le coton. Pour simplifier, nous supposons que la production de ces cultures est séparable des autres du point de vue technologique.
Nous avons donc deux fonctions de production $Y^{m}=F\left(X^{m}, S^{m}\right)$ et $Y^{c}=G\left(X^{c}, S^{c}\right)$ pour le maïs et le coton, respectivement, où les $X_{i}$ sont les quantités d'engrais utilisées pour la culture $i$, les $S_{i}$ sont les surfaces cultivées en culture $i$.

Comme dans Feder (1985), nous supposons que la technologie est à rendement constant: la taille de l'exploitation agricole en tant que telle n'a pas d'effet sur les rendements. À même dose d'engrais par hectare, une exploitation d'un hectare a les mêmes rendements qu'une exploitation de deux hectares. Si les rendements augmentent entre une exploitation d'un et de deux hectares, c'est donc que l'exploitation de deux hectares met plus d'engrais par hectare. Cela permet de ne pas présupposer que, par elle-même, la taille joue sur le rendement. Une telle technologie à rendements constants implique que les fonctions de production peuvent être ramenées à des fonctions de production à l'hectare, production

$y^{m}=\frac{Y^{m}}{S^{m}}=f\left(x^{m}\right)$

et

$y^{c}=\frac{Y^{c}}{S^{c}}=g\left(x^{c}\right)$,

où les $x_{i}$ sont les doses d'engrais par hectare.

La culture du maïs peut utiliser de l'engrais acheté par les producteurs sur le marché. Cependant, pour simplifier, nous considérons que l'ensemble de l'engrais pour la culture du maïs $x^{m} S^{m}$ est fourni par les sociétés cotonnières, et seulement aux producteurs de maiis qui produisent aussi du coton. La quantité fournie par les sociétés cotonnières pour le maïs est plafonnée en fonction de la surface en coton, $x^{m} S^{m} \leq \alpha S^{c}$. Le coton requiert également de l'engrais $x^{c} S^{c}$ fourni par les sociétés cotonnières en proportion de la surface cultivée en coton, $x^{c} \leq \gamma$. En pratique, le seul cas 
pertinent dans le contexte étudié est celui où les producteurs utilisent tout l'engrais disponible et la fonction de production du coton ne dépend alors plus que de la surface en coton puisque le producteur agricole ne choisit pas le degré d'intensification du coton : $y^{c} S^{c}=g(\gamma) S^{c}$. Au Burkina Faso, en pratique, $\alpha=1$ et $\gamma=3$ lorsque les quantités d'engrais sont exprimées en nombre de sacs de $50 \mathrm{~kg}$ de NPK et les surfaces exprimées en hectares. Enfin, la surface cultivée en coton est elle même limitée par négociation au sein des groupements de producteurs de coton qui contractent le crédit. L'enjeu de cette limitation est d'éviter de cultiver de trop grandes surfaces de coton en obtenant un crédit trop important par rapport à la capacité des ménages à le cultiver suffisamment bien pour rembourser le crédit. Mais il s'agit d'une contrainte floue et nous n'en tenons pas compte dans ce modèle. Enfin, nous supposons que la production de coton n'est possible qu'à partir d'une certaine superficie. Les plus petites exploitations ne font que du maïs pour nourrir leur famille sans prendre le risque de produire une culture de rente et sans acheter d'engrais. À partir d'une certaine taille, elles commencent à semer du coton parallèlement à leur maïs. Cette hypothèse correspond à une réalité empirique (figure 4).

\section{Le problème général}

Le programme du producteur s'écrit :

$$
\begin{aligned}
\max _{x^{m}, x^{c}, S^{m}, S^{c}} \pi & =p^{c} y^{c} S^{c} \\
& +p^{m} y^{m} S^{m} \\
& -w\left(S^{c} x^{c}+S^{m} x^{m}\right)
\end{aligned}
$$

$$
\begin{aligned}
& S^{c}+S^{m} \leq S \\
& \left(S^{m}-\underline{S}^{m}\right) S^{c} \geq 0 \\
& x^{m} S^{m} \leq \alpha S^{c} \\
& x^{c} S^{c} \leq \gamma S^{c} \\
& y^{m}=f\left(x^{m}\right) \\
& y^{c}=g\left(x^{c}\right)
\end{aligned}
$$

La variable $w$ est le prix de marché de l'engrais supposé identique au prix de fourniture de l'engrais par les sociétés cotonnières, $S$ est la surface cultivable du ménage.

La deuxième contrainte traduit le passage de l'agriculture de subsistance à la coexistence coton-maïs. Le seuil $\underline{S}^{m}$ est la surface de maïs minimale pour commencer à faire du coton. Si $S^{m} \geq \underline{S}^{m}$ alors $S_{c}$ peut être strictement positif, mais si $S^{m}<\underline{S}^{m}$, on a nécessairement $S^{c}=0$.

Les troisième et quatrième contraintes sont les contraintes sur les engrais fournis par la Sofitex.

Le lagrangien s'écrit :

$$
\begin{aligned}
& L\left(x^{m}, x^{c}, S^{m}, S^{c}\right)=p^{c} y^{c} S^{c}+p^{m} y^{m} S^{m} \\
& -w\left(S^{c} x^{c}+S^{m} x^{m}\right)+\lambda\left(S-S^{c}-S^{m}\right) \\
& +\eta\left(S^{m}-\underline{S}^{m}\right) S^{c}+\mu\left(\alpha S^{c}-x^{m} S^{m}\right) \\
& +\rho\left(\gamma S^{c}-x^{c} S^{c}\right)
\end{aligned}
$$

Avec

$$
\begin{aligned}
& \lambda \geq 0 ;\left(S-S^{c}-S^{m}\right) \geq 0 ; \lambda\left(S-S^{c}-S^{m}\right)=0 \\
& \eta \geq 0 ;\left(S^{m}-\underline{S}^{m}\right) S^{c} \geq 0 ; \eta\left(S^{m}-\underline{S}^{m}\right) S^{c}=0 \\
& \mu \geq 0 ; \alpha S^{c}-x^{m} S^{m} \geq 0 ; \mu\left(\alpha S^{c}-x^{m} S^{m}\right)=0 \\
& \rho \geq 0 ; \gamma S^{c}-x^{c} S^{c} \geq 0 ; \rho\left(\gamma S^{c}-x^{c} S^{c}\right)=0
\end{aligned}
$$

La dérivation de ce programme, respectivement par rapport à $x^{m}, x^{c}, S^{m}$ et $S^{c}$, donne les conditions de premier ordre :

$$
\begin{aligned}
& p^{m} S^{m} f^{\prime}-w S^{m}-\mu S^{m}=0 \\
& p^{c} S^{c} g^{\prime}-w S^{c}-\rho S^{c}=0 \\
& p^{m} y^{m}-w x^{m}-\lambda+\eta S^{c}-\mu x^{m}=0 \\
& p^{c} y^{c}-w x^{c}-\lambda+\eta\left(S^{m}-\underline{S}^{m}\right)+\mu \alpha+ \\
& \rho\left(\gamma-x^{c}\right)=0
\end{aligned}
$$

Nous étudions le cas le plus général au Burkina Faso où toute la surface est utilisée, $\lambda>0$ et $S^{m}=S-S^{c}$ et la quantité d'engrais fournie par la Sofitex est limitante pour le coton $\rho>0 ; \gamma S^{c}-S^{c} x^{c}=0$ et pour le maïs, $\mu>0$ et $x^{m}=\alpha \frac{S^{c}}{S^{m}}$. Le problème devient : 
RECHERCHE

Yves Gérard BAZIE et al.

$$
\begin{aligned}
& p^{m} f^{\prime}-w-\mu=0 \\
& \left(p^{c} g,-w-\rho\right) S^{c}=0 \\
& p^{m} y^{m}-w \alpha \frac{S^{c}}{S^{m}}-\lambda+\eta S^{c} \\
& \quad-\mu \alpha \frac{S^{c}}{S^{m}}=0 \\
& p^{c} y^{c}-w x^{c}-\lambda+\eta\left(S^{m}-\underline{S}^{m}\right)+\mu \alpha=0
\end{aligned}
$$

La résolution dépend essentiellement de la contrainte de subsistance.

\section{La contrainte de subsistance est non contraignante}

Si la contrainte de subsistance n'est pas contraignante, cela signifie que $S^{m}>S^{m}$, $S^{c}>0$ et $\eta=0$. La différence (9) - (10), en substituant $S^{c}$ par $\gamma$, donne :

$$
\begin{gathered}
p^{m} y^{m}-p^{c} y^{c}-w\left(\alpha \frac{s^{c}}{s^{m}}-\gamma\right) \\
-\mu \alpha\left(1+\frac{s^{c}}{s^{m}}\right)=0
\end{gathered}
$$

On introduit la valeur de $\mu$ donnée par l'équation (7).

$$
\begin{aligned}
p^{m} y^{m} & -p^{c} y^{c}-w\left(\alpha \frac{s^{c}}{s^{m}}-\gamma\right) \\
& -\left(p^{m} f^{\prime}-w\right) \alpha\left(1+\frac{s^{c}}{s^{m}}\right)=0
\end{aligned}
$$

Soit :

$$
\begin{aligned}
p^{m} y^{m} & -p^{c} y^{c}+w(\alpha+\gamma) \\
& -p^{m} f^{\prime} \alpha\left(1+\frac{s^{c}}{s^{m}}\right)=0
\end{aligned}
$$

Nous cherchons à exprimer la part de surface en coton $S^{c} / S$ en utilisant $S^{m}=S-S^{c}$ :

$\left[p^{m} y^{m}-p^{c} y^{c}+w(\alpha+\gamma)\right.$

$\left.-p^{m} f^{\prime} \alpha\right]\left(S-S^{c}\right)=p^{m} f^{\prime} \alpha S^{c}$

Qui peut s'écrire :

$\frac{S c}{S}=\frac{p^{m} y^{m}-p^{c} y^{c}+w(\alpha+\gamma)-p^{m} f^{\prime} \alpha}{p^{m} y^{m}-p^{c} y^{c}+w(\alpha+\gamma)}$

Ceci définit une part de surface optimale en coton qui ne dépend pas de $S$. En effet, appelons $\theta=S^{c} / S$ la part de surface en coton, on a alors

$x^{m}=\frac{\alpha S^{c}}{S-S^{c}}=\alpha \theta /(1-\theta)$, donc la fonction de production peut se redéfinir en fonction de $\theta: y^{m}=f\left(x^{m}\right)=h(\theta)$. On se souvient par ailleurs que $y^{c}=g(\gamma)$. L'optimum ci-dessus se réécrit :

$$
\theta^{*}=1-\frac{\alpha p^{m} h^{\prime}\left(\theta^{*}\right)}{p^{m} h\left(\theta^{*}\right)-p^{c} g(\gamma)+w(\alpha+\gamma)}
$$

La part de surface en coton ne dépend pas de $S$, donc l'engrais utilisé sur le coton ne dépend pas de $S$, donc le rendement du maïs ne dépend pas de $S$.

Si les contraintes sur les engrais ne sont pas saturées, il est clair que l'accroissement de la surface totale n'augmente pas non plus le rendement du maïs puisqu'il était déjà à l'optimum. En revanche, dans le cas où la contrainte de sécurité alimentaire est contraignante, $\eta>0$ et $S^{m}=S^{m}$ apporte une différence.

\section{La contrainte de subsistance s'exerce mais la production de coton est possible}

Ce cas se définit par $\eta>0 ; S^{m}=S^{m}$; $S^{c}>0$. Lorsque le ménage dispose d'une surface suffisante pour nourrir sa famille, il consacre le reste de sa surface à la culture du coton. C'est le cas le plus général empiriquement ( $c f$. figure 4). Lorsque la surface totale dépasse 3 ha, la pente de la courbe est proche de 1 , ce qui signifie que presque tout accroissement de surface au-delà de 3 ha est consacré au coton.

Dans ce cas, $\theta$ augmente avec $S$ puisque $\theta=S^{c} / S=\left(S-S^{m}\right) / S=1-S^{m} / S$.

Comme $x^{m}=\alpha \theta /(1-\theta)$, également croissant en $\theta, y^{m}=f\left(x^{m}\right)$ est donc croissant en $S$.

Même si la surface de maïs n'augmente pas, la production de maïs augmente car la surface en maïs reçoit maintenant de l'engrais.

\section{La contrainte de subsistance empêche la production de coton}

Dans cette situation, où la surface cultivée est faible, le producteur ne produit que du maïs sans intrant. Nous avons alors $\eta>0$; $S^{c}=0 ; S^{m}<S^{m}$. Le rendement du maïs est 
constant et indépendant de la surface cultivée. Cette situation se produit jusqu'à ce que la surface cultivée atteigne $S^{m}$, à partir de laquelle le producteur commence à faire du coton.

\section{Illustration de la solution globale}

La figure 3 représente l'effet théorique d'un accroissement de la surface cultivée sur les choix du producteur en matière de surface en coton, de dose d'engrais sur le maïs, et donc de rendement.

Dans la partie gauche des trois schémas correspondant à ces choix, lorsque $S<S^{m}$, la surface totale est trop faible pour que le ménage puisse produire du coton et toute sa surface est consacrée au maïs. Il n'utilise pas d'engrais et le rendement du maïs ne varie pas avec la surface totale.

Dans la partie intermédiaire où $S^{m}<S<S^{m}+S^{c}$, le ménage consacre toute sa surface supplémentaire à la culture du coton. La pente de la droite sur ce segment est égale à 1 , et $S^{c}$ est la surface de coton minimale à partir de laquelle le rapport des surfaces devient optimal (et la surface de maïs recommence à augmenter). L'idée est que les ménages concernés rattrapent

Figure 3. Solution globale

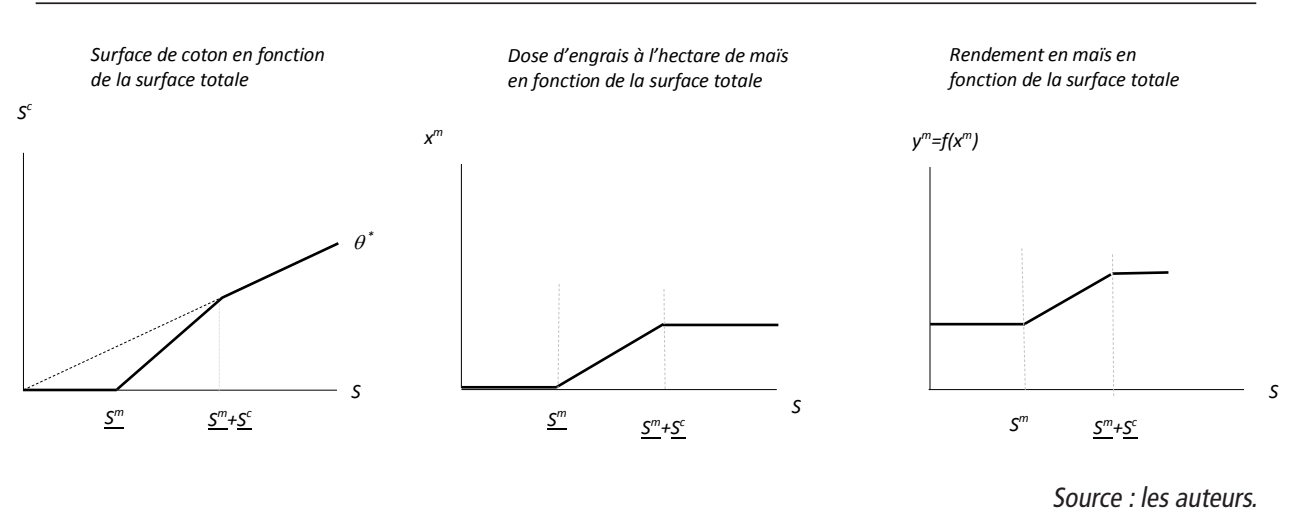

Figure 4. Observations de la surface de coton en fonction de la surface totale, année 2010

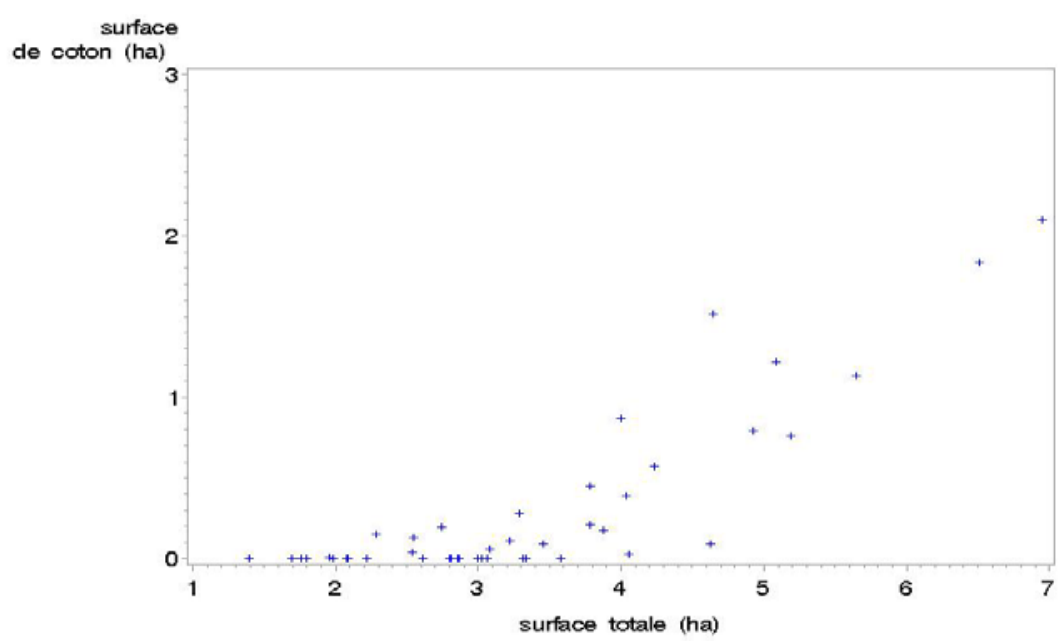

Source : les auteurs. 
RECHERCHE

Yves Gérard BAZIE et al.

l'optimum économique le plus «vite» possible, or cet optimum se caractérise dans notre modèle par une plus grande proportion de coton $\left(\theta^{*}\right)$. L'utilisation d'engrais pour le maïs augmente avec $S$ car $S^{c}$ augmente plus vite que $S$ en valeur relative (on rappelle que $x^{m}=\frac{\alpha S^{c}}{S-S^{c}}$ ). Le rendement du maïs augmente avec $x^{m}$.

Dans la partie de droite, $S>S^{m}+S^{c}$, le ménage a maintenant « retrouvé » sa proportion optimale de coton/maïs et les deux surfaces augmentent en proportion fixe. La dose d'engrais du maïs devient constante et le rendement du maïs aussi.

\section{Effet attendu}

En théorie, l'effet attendu de la surface cultivée sur la productivité du maïs devrait être nul pour les petites surfaces, positif pour les moyennes surfaces, et nul pour les surfaces plus élevées. Nous constatons sur la figure 4 que ce dernier cas n'a apparemment pas une grande importance empirique, mais si c'était le cas, en linéarisant l'effet de la surface sur la productivité, l'effet attendu moyen sur l'ensemble de la population serait positif.

Il est possible de décomposer cet effet attendu en sous-effets qui nous aideront à donner du crédit à notre thèse. En particulier, il est attendu que la productivité du maiis soit plus élevée lorsque le producteur produit aussi du coton, et lorsque la surface de coton augmente, ce qui en théorie va avec l'accroissement de la surface totale.

\section{Estimation empirique}

Nous cherchons à tester l'effet de la surface totale sur la productivité des céréales, et en particulier du maïs, dans les systèmes de production agricole du Burkina Faso. Sur la période 1995-2012, nous avons mobilisé des données ménages, des données de pluviométrie et des données de prix.

La plupart des articles sur la relation entre taille des exploitations agricoles et productivité se situent au niveau de l'exploitation agricole, c'est-à-dire toutes cultures confondues. Quelques articles toutefois se situent au niveau des parcelles et portent donc de manière spécifique sur certaines cultures, comme le riz à Madagascar (Barrett, 1996) ou bien le maïs en Zambie (Kimhi, 2006). Dans cet article, nous analysons l'effet de la surface totale sur la productivité globale des céréales ainsi que sur la productivité partielle du maïs, du mil et du sorgho.

\section{Estimation d'un modèle de panel}

\section{Des observations au niveau provincial}

Les données ménages portent sur des décisions individuelles, et sont disponibles pour environ 4500 ménages (une observation par ménage et par an). Les données de pluviométrie et les données de prix des produits agricoles sont quant à elles disponibles au niveau provincial, sur une base de 45 provinces (une observation par an et par province pour la pluviométrie, une observation par mois et par province pour les prix). Nous avons procédé à une agrégation des données ménages au niveau provincial pour mettre en correspondance ces données aux autres types de données. Chaque variable individuelle (surface, rendement notamment mais également les autres types de données) est ramenée à sa moyenne provinciale. L'enquête ménage réalisée chaque année s'appuie sur un design de randomisation à deux étapes. Dans un premier temps, les villages sont choisis aléatoirement par province, leur nombre dépendant de la population de chaque province. Dans un second temps, les ménages sont choisis aléatoirement au sein des villages. Cette randomisation en deux temps fait que les ménages enquêtés sont représentatifs des ménages agricoles au niveau de la province et que nous pouvons avoir recours à des moyennes provinciales pour analyser la diversité des décisions agricoles. 
Il est certain que l'agrégation des données ménages au niveau provincial correspond à une perte d'information dans la mesure où l'agrégation « écrase » la variabilité des comportements des ménages au sein des provinces et privilégie une analyse de la variabilité des comportements individuels d'une province à une autre. Nous obtenons ainsi un panel dans lequel les variables comme les prix et la pluviométrie changent avec la même fréquence que la variable endogène. Si ce n'était pas le cas, les estimateurs pourraient être biaisés, du fait de la stabilité de certaines variables exogènes. L'échantillonnage réalisé par le ministère de l'Agriculture du Burkina Faso nous assure de la représentativité des données individuelles collectées au niveau provincial et nous permet donc de transposer en toute confiance les mécanismes individuels décrits dans le modèle théorique à l'échelle provinciale. D'autres papiers modélisent des décisions individuelles à partir de données agrégées, obtenues à partir d'un échantillon large et choisi de manière aléatoire (Balestra et Nerlove, 1966).

La surface moyenne d'un ménage à l'échelle d'une province est probablement moins exogène que la surface individuelle d'un ménage dans une province. En effet, elle peut résulter de trajectoires similaires de nombreux producteurs qui dépendent notamment du rendement espéré dans la province. Par exemple, les conditions pédoclimatiques favorables aux hauts rendements risquent de favoriser l'accroissement de la population locale et donc de réduire la surface disponible par ménage. Les provinces plus urbaines risquent d'exposer les producteurs à une demande plus forte en céréales, et donc de stimuler les rendements, mais aussi les accroissements de surface s'il reste des terres disponibles. La spécialisation des activités, par exemple liée au climat ou à l'existence de microfinance, peut aussi impacter la surface cultivée. Ces éléments constituent de l'hétérogénéité inobservée corrélée à la surface cultivée conduisant à remettre en cause l'hypothèse d'indépendance conditionnelle des aléas, nécessaire en cas de régression linéaire.

L'exploitation des données de panel permet de corriger en partie ce problème d'hétérogénéité inobservée corrélée à la surface cultivée. Un panel à effet fixe permettrait de corriger ce problème si l'hétérogénéité inobservée était constante, mais pas si c'est une trajectoire liée à l'urbanisation ou à toute autre dynamique. Pour cela, il nous faut estimer un panel dynamique corrigeant, autant que possible, l'effet que pourrait avoir le rendement passé sur la surface cultivée.

\section{Un modèle de panel avec effet retard}

L'estimation d'un modèle statique n'est pas très performante parce que les effets géographiques dominent trop nettement les effets temporels. Si nous comparons les provinces entre elles, la relation entre le prix et la production est négative, car les régions les plus productives engendrent des prix en moyenne plus faibles. Afin que le prix soit une variable exogène de la production, nous utilisons le prix observé avant la décision de produire, comme nous comparons la productivité après une année de faible prix et la productivité de cette région après une année de prix élevé. Nous nous attendons alors à une relation positive entre le prix et la productivité.

L'équation (17) qui suit est la version linéarisée de notre modèle théorique. Dans le modèle théorique, le rendement du maïs dépend : de la surface totale, de la surface minimale avant de faire du coton, $S^{m}$, du prix du coton, du prix du maïs, et du prix des engrais. Nous ne disposons pas de données sur le prix des engrais, mais les prix sont uniformes et varient peu dans le temps. Nous ne disposons pas non plus de données sur le prix du coton, ce qui est plus problématique parce qu'il varie 
d'une année à l'autre, et c'est une source d'imprécision dans notre estimation. Cela étant dit, le prix du coton joue sur le rendement du maïs pour les surfaces les plus grandes (partie droite de la figure 3, cf. équation 16), qui n'est empiriquement pas prépondérante. Par ailleurs, la contrainte de surface minimale avant de faire du coton est remplacée par une dummy valant 1 si le ménage produit du coton, et le cumul pluviométrique est une variable implicite de la fonction de production $y^{m}=f\left(x^{m}\right)$.

$y_{i j t}=a_{0}+a_{1} S_{i t}+a_{2} \mathrm{I}_{\text {coton }, i t}+a_{3} \mathrm{PLUIE}_{i t}$ $+a_{4} p_{j t-1}+Z_{i t} b+\mu_{i}+v_{t}+\varepsilon_{i j t}$

où $Z_{i t}$ est un vecteur de variables individuelles exogènes et $y_{i j t}$ est la productivité moyenne par ha des céréales de type $j$ dans la province $i$ pour l'année $t$. La variable $S_{i t}$ est la surface totale disponible pour l'agriculteur moyen de la province $i$ pour l'année $t$.

De par les problèmes d'endogénéité potentiels entre les variables explicatives et les rendements, nous introduisons un effet retard dans le modèle que nous estimons comme suit :

$y_{i j t}=b_{0}+b_{1} y_{i t-1}+b_{2} S_{i t}+b_{3} \mathrm{I}_{\text {coton, }, i t}$

$+b_{4}$ PLUIE $_{i t}+b_{5} p_{j t-1}+Z_{i t} \gamma+\chi_{i j t}$

La prise en compte d'un effet retard, par l'introduction d'une variable de rendement décalée, permet de corriger les problèmes d'endogénéité pouvant provenir d'un ajustement progressif de la surface cultivée aux rendements espérés. Par exemple, le fait qu'une province ait une productivité élevée la rend plus attractive, ce qui peut aboutir à accroître la pression foncière et réduire la surface disponible par actif agricole. Dans ce cas, la qualité des sols pourrait expliquer à la fois les surfaces et les rendements et serait alors une source d'hétérogénéité inobservée causant un biais dans le coefficient $a_{1}$. En principe, les effets fixes d'un panel permettent de capturer cette hétérogénéité et corriger le biais, seulement si elle est constante, or ce n'est vraisemblablement pas le cas ici. Non seulement la qualité du sol n'est pas constante en fonction des trajectoires locales, mais d'autres facteurs affectent à la fois les surfaces et les rendements d'une manière non constante (spécialisation, urbanisation, etc.). Pour cette raison, il est préférable d'estimer un panel avec variable retardée, destiné à capturer les effets de l'histoire passée de la région qui expliquent les relations entre surfaces et rendement autres que la relation que nous cherchons à mettre en évidence.

Nous utilisons l'estimateur de la méthode des Moments Généralises (GMM) de Arellano et Bond (1991). Cette méthode est adaptée à la prise en compte de cet effet retard dans une structure de panel. Elle permet d'instrumenter : la variable surface par des différences interannuelles passées, les valeurs en niveau passées de cette même variable, ainsi que de la pluviométrie exogène avec certitude.

Après estimation, il s'avère que le rendement est très peu déterminé par le rendement passé (les données montrent un comportement instable des rendements, très déterminés par les pluies et par les prix, eux-mêmes très volatils au Burkina). Mais l'enjeu d'utiliser la méthode des GMM, en cas d'hétérogénéité inobservée non constante, demeure pour l'instrumentation de la surface.

\section{Présentation des variables}

Les variables expliquées dans notre modèle économétrique sont le rendement du maïs, du sorgho et du mil, principales céréales produites au Burkina Faso. Les variables explicatives retenues sont: (1) le rendement provincial moyen de l'année passée des céréales ; (2) la surface totale cultivée de l'exploitation provinciale moyenne, cette surface pouvant être décomposée en surface cultivée hors coton et en surface cultivée en coton afin de déterminer si 
l'effet de la surface sur le rendement est plus particulièrement lié à la production de coton (test empirique du mécanisme explicatif présent dans notre modèle théorique); (3) une indicatrice de l'existence du coton dans les systèmes de production provinciaux (qui prend la valeur 1 s'il y a du coton produit dans la province et 0 sinon); (4) le cumul pluviométrique de l'année en cours dans la province ; et (5) la moyenne provinciale des prix des céréales au cours de l'année précédente. Les variables (2) et (3) nous permettent de tester directement la validité empirique des effets théoriques attendus.

La dynamique de production passée a pu impacter le prix $P_{j, t-1}$, ce qui pourrait rendre cette variable endogène. Pour purger le coefficient associé à cette variable, $b_{5}$, des effets passés de la production sur les prix d'équilibre, on introduit la variable de rendement décalé, $y_{i j t-1}$, qui est censée capturer les effets de la dynamique passée.

Si $b_{2}>0$, c'est que $\frac{y^{\prime}}{d S}>0$ (principal effet attendu).

Les effets fixes provinces traduisent le fait que les rendements entre provinces ne sont pas comparables puisque des variables non observables comme la qualité des sols et la pression démographique diffèrent. L'estimation en panel dynamique ne considère pas directement ces effets fixes mais intègre cette hétérogénéité inobservée dans l'estimation des paramètres.

\section{Présentation des données}

L'estimation du modèle dynamique que nous venons de présenter se fait par la mobilisation de trois bases de données originales.

La première est issue de l'enquête permanente agricole menée par le ministère de l'Agriculture du Burkina Faso chaque année auprès d'un échantillon représentatif d'environ 4500 ménages agricoles couvrant l'ensemble des 45 provinces du pays. Les données sont collectées chaque année en novembre, après les récoltes des cultures pluviales. La base de données utilisée correspond à la fusion de 18 bases de données annuelles et à la constitution de moyennes provinciales à partir des données des ménages. Le tableau 1 présente les principales variables utilisées.

La deuxième base de données utilisée est celle des cumuls pluviométriques annuels

Tableau 1. Description des exploitations agricoles étudiées (moyennes provinciales sur la période 1995-2012)

\begin{tabular}{lcccc}
\hline Variable & Moyenne & Écart-Type & Min & Max \\
\hline Nombre de membres du ménage & 10,5 & 1,7 & 5,9 & 17,4 \\
Nombre d'actifs dans le ménage & 6,7 & 1,3 & 2,9 & 11,1 \\
Surface en maïs (ha) & 0,4 & 0,6 & 0,0 & 2,7 \\
Surface en mil (ha) & 1,2 & 0,9 & 0,0 & 5,3 \\
Surface en sorgho (ha) & 1,4 & 0,7 & 0,0 & 3,6 \\
Surface en coton (ha) & 0,4 & 0,7 & 0,0 & 3,8 \\
Surface totale (ha) & 4,1 & 1,5 & 1,1 & 10,2 \\
Nombre de têtes de bétail & 6,3 & 3,8 & 0,3 & 27,8 \\
Nombre de charrues & 1,1 & 0,5 & 0,0 & 2,8 \\
Rendement de maïs (kg/ha) & 1119 & 435 & 60 & 2960 \\
Rendement de mil (kg/ha) & 734 & 221 & 148 & 1444 \\
Rendement de sorgho (kg/ha) & 888 & 244 & 185 & 1654 \\
\hline
\end{tabular}

Note : 62 \% des ménages enquêtés produisent du coton. 


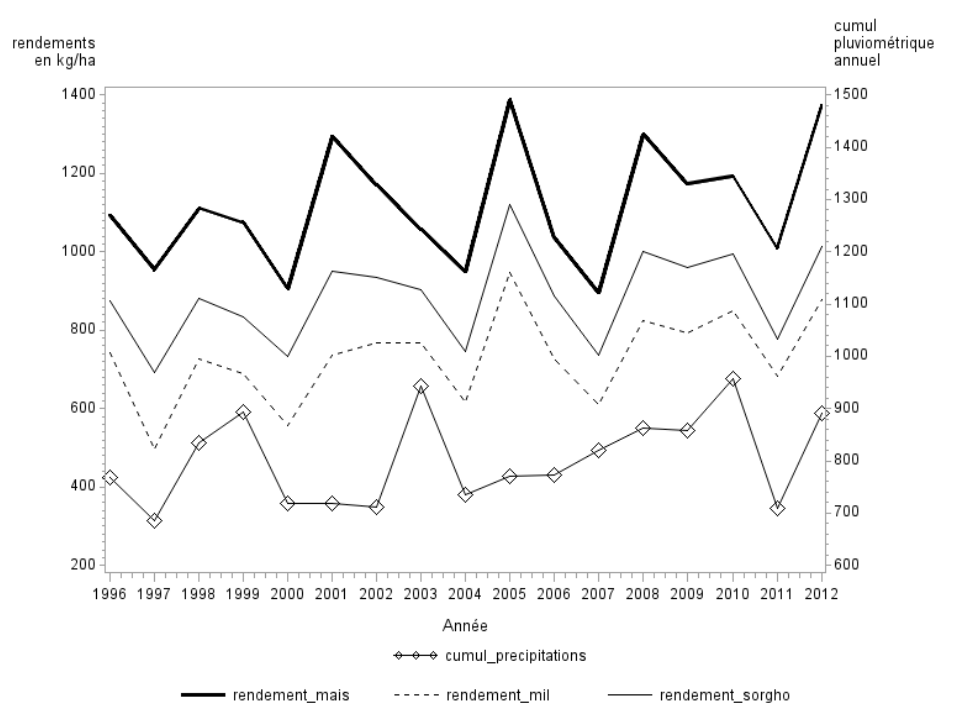

Source : les auteurs.

au niveau des 45 provinces étudiées, fournis par la direction de la Météorologie. L'évolution des cumuls pluviométriques annuels depuis 1980 est présentée par la figure 9 en annexe, pour trois zones climatiques distinctes : la zone sahélienne (plus au nord), la zone soudano-sahélienne et la zone soudanienne (plus au sud). Les cumuls pluviométriques sont plus importants dans les zones situées au sud. La figure 5 met en parallèle l'évolution des précipitations moyennes et des rendements moyens de maïs, mil et sorgho au Burkina Faso.

Nous voyons que les cumuls pluviométriques varient fortement d'une année à l'autre et que sur le temps long il pleut de plus en plus (tendance positive). Par ailleurs, les rendements sont corrélés positivement aux cumuls pluviométriques.

La troisième base de données utilisée est celle des prix des céréales publiés chaque mois par la société nationale de gestion du stock de Securit au niveau des 45 provinces étudiées. Les prix utilisés dans les régressions sont les moyennes annuelles non pondérées des prix relevés sur la période de janvier à juillet, c'est-àdire avant l'installation des cultures afin d'éviter les problèmes de causalité inverse et d'endogénéité. Les céréales sont des cultures pluviales au Burkina Faso, leur saison de culture correspond à la saison des pluies et s'étend de juillet à octobre. La figure 6 met en parallèle l'évolution des prix courants et des rendements moyens de maïs, mil et sorgho au Burkina Faso.

Les prix moyens du maïs, du mil et du sorgho suivent des évolutions comparables, ce qui indique que globalement les productions sont substituables. Les prix moyens varient fortement d'une année à l'autre. Nous voyons que les rendements sont positivement corrélés dans le temps aux prix courants, indice que des prix élevés sur la période pré-culturale (de janvier à juillet) sont des incitations pour les producteurs à intensifier leurs productions.

\section{Résultats empiriques}

L'estimation du modèle dynamique fournit les résultats suivants, consignés dans le tableau 2. Quatre spécifications sont 
Figure 6. Évolution des prix courants et des rendements moyens nationaux de céréales au Burkina Faso

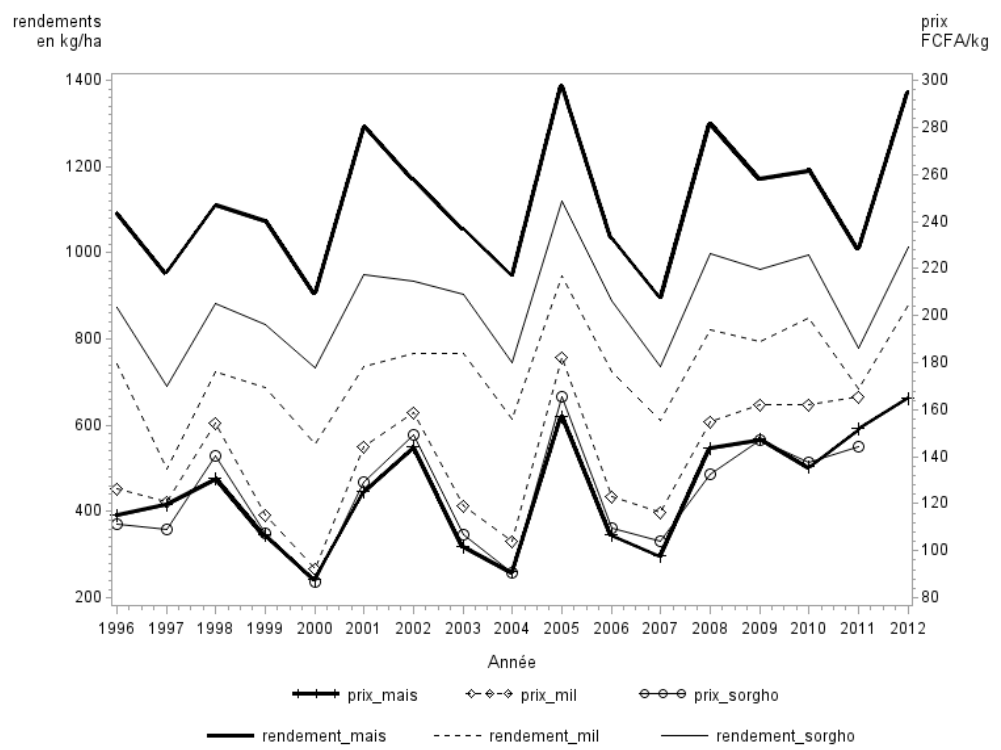

Source : les auteurs.

Tableau 2. Estimation des effets de la taille des exploitations sur les rendements par la méthode des moments généralisés de Arrellano et Bond

\begin{tabular}{lcccc}
\hline & Céréales (1) & Maïs (2) & Mil (3) & Sorgho (4) \\
\hline Constante & $18,16^{* * *}$ & 5,63 & $16,80^{* * *}$ & $10,06^{*}$ \\
& $(3,32)$ & $(0,58)$ & $(3,42)$ & $(1,87)$ \\
Rendement décalé & 0,00 & $-0,14$ & $-0,09$ & $-0,06$ \\
& $(-0,01)$ & $(-1,51)$ & $(-1,47)$ & $(-1,01)$ \\
Surface cultivée & $34,24 * * *$ & $45,43^{* *}$ & $22,60^{* *}$ & $20,27^{*}$ \\
& $(3,09)$ & $(2,46)$ & $(2,32)$ & $(1,92)$ \\
Existence de coton & $190,97^{*}$ & $588,55^{* * *}$ & 144,58 & $205,72^{*}$ \\
& $(1,48)$ & $(2,63)$ & $(1,30)$ & $(1,65)$ \\
Cumul pluviométrique & $0,25^{* * *}$ & 0,02 & $0,28^{* * *}$ & $0,32^{* * *}$ \\
& $(3,32)$ & $(0,14)$ & $(4,08)$ & $(4,34)$ \\
Prix du maïs & 2,16 & $6,72^{* * *}$ & $4,88^{* * *}$ & $5,75^{* * *}$ \\
& $(1,19)$ & $(2,12)$ & $(2,94)$ & $(3,28)$ \\
Prix du mil & $12,92^{* * *}$ & $13,20 * * *$ & 2,50 & 3,04 \\
& $(4,70)$ & $(2,82)$ & $(1,03)$ & $(1,14)$ \\
Prix du sorgho & $-12,74^{* * *}$ & $-15,70^{* *}$ & $-4,64$ & $-5,43$ \\
& $(-3,54)$ & $(-2,52)$ & $(-1,44)$ & $(-1,55)$ \\
Nombre de provinces & 38 & 38 & 38 & 38 \\
Longueur des séries temporelles & 16 & 16 & 16 & 16 \\
\hline
\end{tabular}

Notes : $t$-test entre parenthèses; ${ }^{*}(p<0,1) ;{ }^{* *}(p<0,05) ;{ }^{* *}(p<0,01)$ 
présentées, selon que la variable expliquée est le rendement moyen des céréales (1), du maïs (2), du mil (3), ou du sorgho (4).

Les provinces comportant des données manquantes sur les prix n'ont pas été prises en compte dans l'analyse de panel dynamique par le logiciel. Nous avons choisi de ne pas remplacer les données manquantes, d'où la perte de sept provinces dans notre analyse économétrique.

\section{Interprétation}

Le rendement moyen en céréales de l'année passée n'a pas d'effet significatif sur le rendement moyen en céréales de cette année, ce qui traduit avant tout la forte variabilité des rendements observés d'une année sur l'autre.

La surface cultivée a un effet positif sur les rendements des trois céréales étudiées. En moyenne, l'augmentation d'un hectare de surface agricole cultivée se traduit par des gains de rendement de $20 \mathrm{~kg} / \mathrm{ha}$ pour le sorgho, de $23 \mathrm{~kg} / \mathrm{ha}$ pour le mil et de $45 \mathrm{~kg} / \mathrm{ha}$ pour le maïs. Ce résultat confirme l'existence d'une relation positive entre taille des exploitations agricoles et productivité des céréales au Burkina Faso.

En plus de l'effet positif des surfaces cultivées, le fait de produire du coton augmente significativement les rendements moyens des céréales. En moyenne, pour une même surface cultivée globale, les producteurs de coton ont des rendements plus élevés d'environ $200 \mathrm{~kg} / \mathrm{ha}$ pour les céréales et $600 \mathrm{~kg} / \mathrm{ha}$ pour le maïs, ce qui semble donner du crédit au mécanisme suggéré dans la partie théorique du papier.

Nous trouvons un effet positif significatif du cumul pluviométrique sur les rendements moyens des céréales, du mil et du sorgho. Cela peut être expliqué par le fait que les productions de mil et de sorgho sont plus directement dépendantes de la réalisation de la pluie dans la mesure où elles ne dépendent que de la pluie (et de la maind'œuvre qui ne varie pas considérablement d'une année à une autre) quand le maïs dépend de la pluie et de l'application d'engrais chimique. Les effets des prix du maïs, du mil et du sorgho sont à rapporter au caractère plus ou moins substituable ou complémentaire de ces céréales entre elles. Par exemple, l'effet significatif négatif du prix du sorgho sur les rendements en céréales et plus particulièrement sur les rendements en maiis semble indiquer une substitution du sorgho avec le maïs : si le prix du sorgho augmente, les producteurs vont avoir tendance à chercher de plus importants rendements de sorgho et moins de maïs (réallocation des facteurs). Cet effet de substitution ne s'applique pas pour le mil (céréale moins substituable au maïs) qui est en général fait en plus du maïs et pour laquelle on aurait plutôt une complémentarité (rendement de céréales et de maïs positivement corrélés au prix du mil).

\section{Discussion}

Comme test de robustesse, nous avons estimé trois modèles économétriques dans lesquels les rendements de chaque culture dépendent des prix de la culture en question et non des autres prix. Les résultats sont robustes à une telle spécification, à savoir que la relation entre la surface en coton et le rendement des différentes céréales reste significativement positif. Ces résultats sont présentés dans le tableau 3 en annexe.

Dans notre modèle théorique, le fait de produire du coton et la surface cultivée en coton dépendent de la surface totale, qui est supposée exogène. En réalité, la production de coton dépend probablement de caractéristiques inobservées des producteurs, comme les contraintes de liquidités ou l'aptitude à produire du coton. C'est là une limite de notre estimation statistique. Néanmoins, nous pensons que la production de coton dépend beaucoup de la localisation et de la surface cultivable car les producteurs qui ont une surface cultivable suffisante dans les régions favorables à la culture de coton tendent à produire du 
coton. Si la production de coton est en grande partie déterminée par la possibilité de produire du coton, nous ne pouvons toutefois pas exclure une part d'autosélection dans le choix de produire du coton, due à des facteurs inobservés.

*

La productivité agricole des céréales a augmenté au Burkina Faso au cours des vingt dernières années. Le point de départ de cet article était une observation empirique selon laquelle les plus grandes exploitations sont plus productives en moyenne que les plus petites exploitations ; observation qui va à l'encontre d'un constat empirique généralement établi voulant que la productivité agricole soit plus importante pour les exploitations de petite taille. L'article analyse l'accès à l'engrais facilité via la production de coton comme facteur explicatif de la relation positive entre taille des exploitations et productivité des céréales au Burkina Faso. Le fait de produire du coton garantit aux producteurs de pouvoir accéder à l'engrais chimique sans avoir à en avancer le coût à l'installation des cultures, période de l'année où les contraintes de liquidité sont particulièrement importantes pour les producteurs agricoles (le prix de l'engrais est par la suite déduit du prix d'achat du coton). Un modèle de demande d'engrais inspiré de Feder (1985) est développé pour intégrer la spécificité des systèmes de production agricole du Burkina Faso et notamment l'interdépendance entre les productions de maïs et de coton. Le modèle établit que le rendement du maïs augmente avec la surface totale. La validité empirique de ce modèle est testée par la mobilisation de données ménages, de données de prix et de données pluviométriques sur la période 1995-2012. L'effet positif de la surface totale cultivée sur la productivité des céréales est confirmé, et il est démontré que cet effet est tiré par les surfaces cultivées en coton, ce qui confirme le mécanisme explicatif avancé. Les implications politiques d'un tel résultat sont que l'accessibilité à l'engrais est un levier important des gains de productivité réalisables et constitue donc un enjeu de développement, et de sécurité alimentaire pour le Burkina Faso. Cet accès aux engrais est aujourd'hui fortement limité compte tenu des coûts élevés de transaction et de transport mais surtout dû aux contraintes de liquidité fortes qui pèsent sur les producteurs agricoles et des imperfections du marché du crédit. Des mécanismes nouveaux de subvention du prix des engrais (smart subsidies) sont à l'étude et pourraient se traduire par une diminution du prix de l'engrais au producteur, par une augmentation conséquente du recours à l'engrais et par une amélioration de la productivité agricole des ménages. 


\section{RÉFÉRENCES BIBLIOGRAPHIQUES}

Arellano M., Bond S. (1991). Some tests of specification for panel data: Monte Carlo evidence and an application to employment equations. The Review of Economic Studies, vol. 58, n ${ }^{\circ}$ 2, pp. 277-297.

Balestra P., Nerlove M. (1966). Pooling cross section and time series data in the estimation of a dynamic model: The demand for natural gas. Econometrica, vol. 34, $\mathrm{n}^{\circ} 3$, pp. 585-612.

Bardhan P. K. (1973). Size, productivity, and returns to scale: An analysis of farm-level data in Indian agriculture. Journal of Political Economy, vol. 81, n 6, pp. 1370-1386.

Barrett C. B. (1996). On price risk and the inverse farm size-productivity relationship. Journal of Development Economics, vol. 51, $\mathrm{n}^{\circ} 2$, pp. 193-215.

Barrett C. B., Bellemare M. F., Hou J.Y. (2010). Re-considering conventional explanations of the inverse productivity-size relationship. World Development, vol. 38, $\mathrm{n}^{\circ} 1$, pp. 88-97.

Benjamin D. (1995). Can unobserved land quality explain the inverse productivity relationship? Journal of Development Economics, vol. $46, \mathrm{n}^{\circ} 1$, pp. 51-84.

Berry R. A., Cline W. R (1979). Agrarian structure and productivity in developing countries: a study prepared for the International Labour Office within the framework of the World Employment Programme. Johns Hopkins University Press, 248p.

Bhalla S. S. (1988). Does land quality matter? Theory and measurement. Journal of Development Economics, vol. 29, $\mathrm{n}^{\circ} 1$, pp. 45-62.

Bhalla S. S., Roy P. (1988). Mis-specification in farm productivity analysis: the role of land quality. Oxford Economic Papers, vol. $40, \mathrm{n}^{\circ} 1$, pp. 55-73.

Carter M. R. (1984). Identification of the inverse relationship between farm size and productivity: an empirical analysis of peasant agricultural production. Oxford Economic Papers, vol. 36, $\mathrm{n}^{\circ}$ 1, pp. 131-145.
Carter M. R., Wiebe K. D. (1990). Access to capital and its impact on agrarian structure and productivity in Kenya. American Journal of Agricultural Economics, vol. 72, $\mathrm{n}^{\circ} 5$, pp. 1146-1150.

Chand R., Prasanna P. L., Singh A. (2011). Farm size and productivity: Understanding the strengths of smallholders and improving their livelihoods. Economic and Political Weekly, pp. 5-11.

Collier P., Dercon S. (2014). African Agriculture in 50 Years: Smallholders in a Rapidly Changing World? World Development, Economic Transformation in Africa, $\mathrm{n}^{\circ}$ 63, pp. 92-101.

Dowd-Uribe B. (2014). Engineering yields and inequality? How institutions and agroecology shape Bt cotton outcomes in Burkina Faso. Geoforum, n ${ }^{\circ}$ 53, pp. 161-171.

Eswaran M., Kotwal A. (1986). Access to capital and agrarian production organisation. The Economic Journal, vol. 96, $\mathrm{n}^{\circ} 382$, pp. 482-498.

Feder G. (1985). The relation between farm size and farm productivity: The role of family labor, supervision and credit constraints. Journal of Development Economics, vol. 18, n 2-3, pp. 297-313.

Heltberg R. (1998). Rural market imperfections and the farm size-productivity relationship. Evidence from Pakistan. World Development, vol. 26, n 10, pp. 1807-1826.

Kevane M. (1996). Agrarian structure and agricultural practice: Ty-pology and application to Western Sudan. American Journal of Agricultural Economics, vol. 78, $\mathrm{n}^{\circ} 1$, pp. 236-245.

Kimhi A. (2006). Plot size and maize productivity in Zambia: is there an inverse relationship? Agricultural Economics, vol. 35, $\mathrm{n}^{\circ} 1$, pp. 1-9.

Larson D. F., Otsuka K., Matsumoto T., Kilic T. (2014). Should African rural development strategies depend on smallholder farms? An exploration of the inverse-productivity hypothesis. Agricultural Economics, vol. 45, $\mathrm{n}^{\circ} 3$, pp. 355-367. 
Le Cotty T., Maître d'Hôtel E., Soubeyran R., Subervie J. (2018). Linking risk aversion, time preference and fertilizer use in Burkina Faso. The Journal of Development Studies, vol. 54, n 11 , pp. 1991-2006.

Sawadogo H., Bock L., $\quad$ Lacroix D., Zombre N. P. (2008). Restauration des potentialités de sols degradés à l'aide du zaï et du compost dans le Yatenga (Burkina Faso).
Biotechnologie, Agronomie, Société et Environnement, vol. 12, n 3, pp. 279-290.

Zaibet L. T., Dunn E. G. (1998). Land tenure, farm size, and rural market participation in developing countries: the case of the Tunisian olive sector. Economic Development and Cultural Change, vol. 46, $\mathrm{n}^{\circ} 4$, pp. 831-848. 
RECHERCHE

Yves Gérard BAZIE et al.

\section{ANNEXES}

Figure 7. Relation entre productivité du maïs et surface totale au Burkina Faso (moyennes par province et par an)

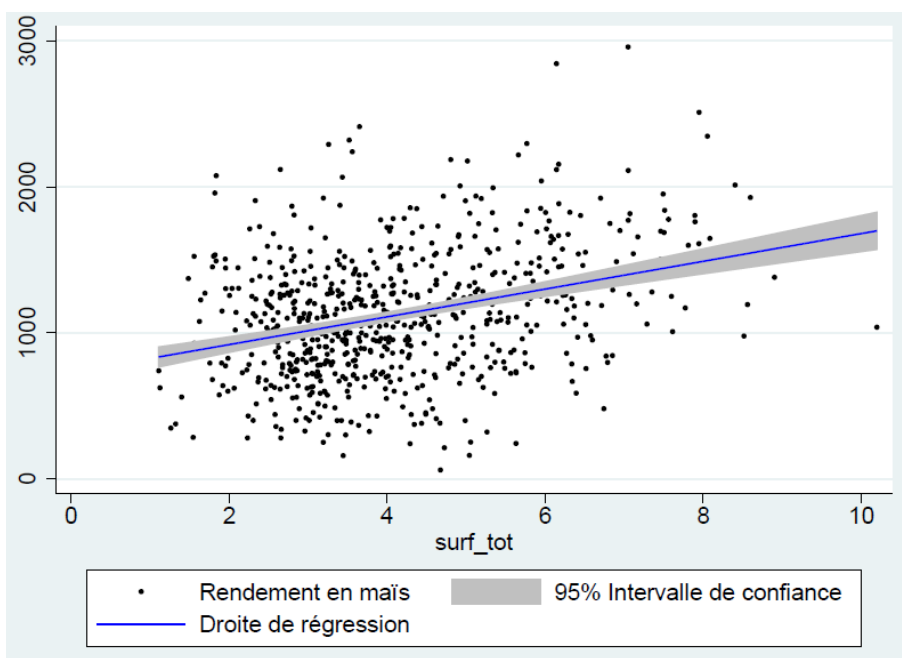

Source : les auteurs.

Figure 8. Évolution de la relation entre productivité des céréales et surface totale au Burkina Faso

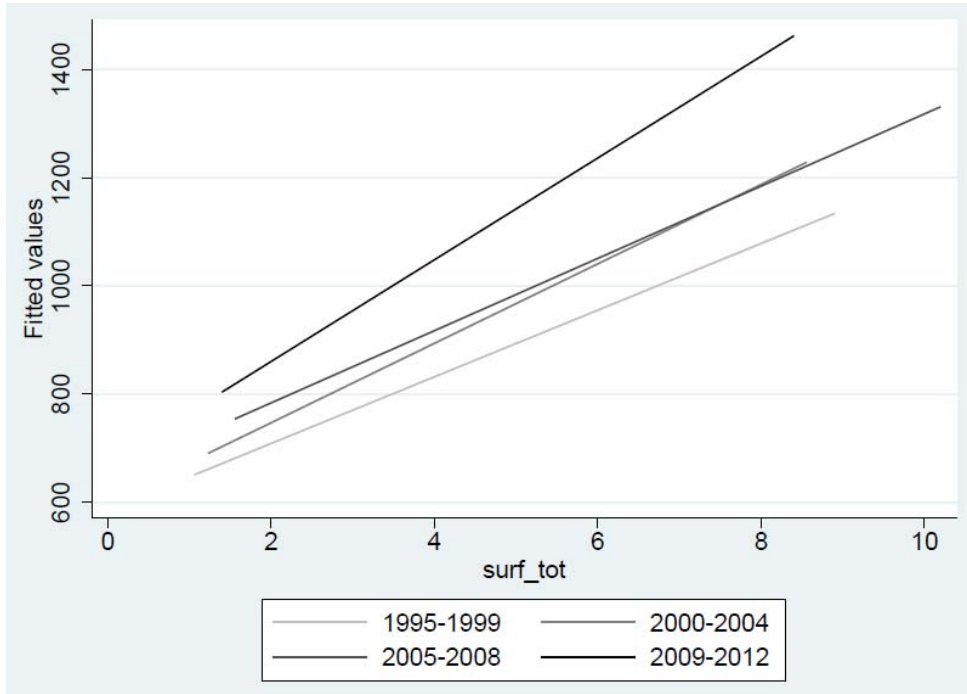

Source : les auteurs. 
Figure 9. Cumul pluviométrique annuel au Burkina Faso (1980-2012)

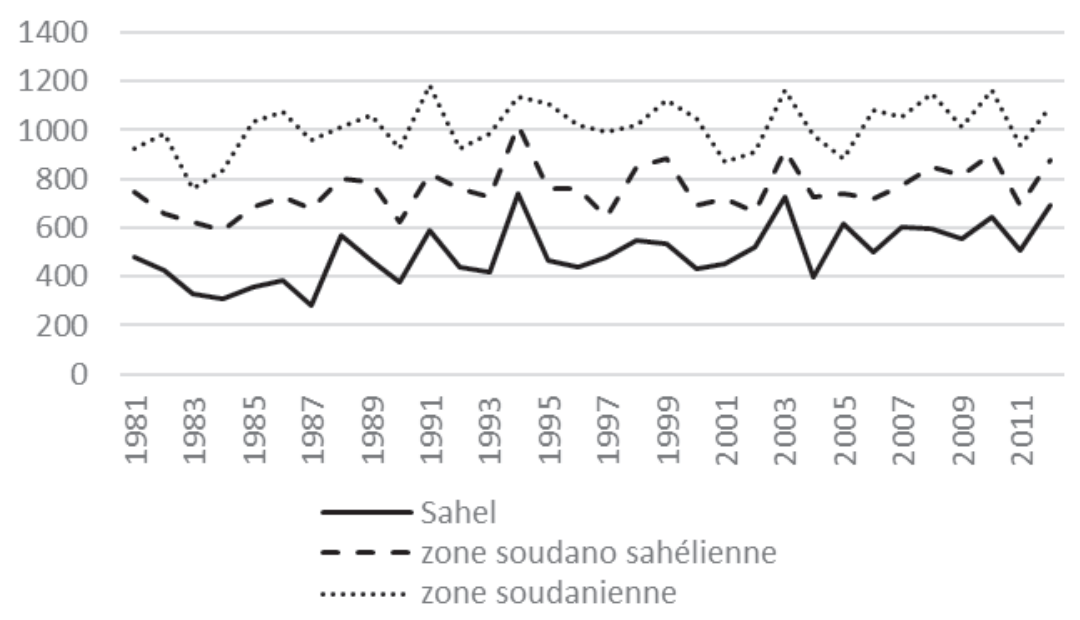

Source : les auteurs.

Tableau 3. Estimations GMM des rendements de chaque culture en fonction du prix de la culture

\begin{tabular}{lccc}
\hline & Maïs & Mil & Sorgho \\
\hline Constante & 9.54 & $14.87 * * *$ & $9.08 *$ \\
& $(0.96)$ & $(3.40)$ & $(1.91)$ \\
Rendement décalé & -0.12 & -0.06 & -0.05 \\
& $(-1.31)$ & $(-1.10)$ & $(-0.93)$ \\
Surface cultivée & $46.44 * * *$ & $27.63 * * *$ & $26.33 * * *$ \\
Existence de coton & $(2.39)$ & $(2.95)$ & $(2.64)$ \\
& $645.35 * * *$ & 132.02 & $199.28 * *$ \\
Cumul pluviométrique & $(2.75)$ & $(1.23)$ & $(1.70)$ \\
& 0.04 & $0.27 * * *$ & $0.32 * * *$ \\
Prix de la céréale & $(0.29)$ & $(4.02)$ & $(4.40)$ \\
& $5.13 * * *$ & $2.55 * * *$ & $3.53 * * *$ \\
& $(6.60)$ & $(7.08)$ & $(8.53)$ \\
\hline
\end{tabular}

Notes : $t$-test entre parenthèses; ${ }^{*}(p<0,1) ;{ }^{* *}(p<0,05) ;{ }^{* * *}(p<0,01)$ 